\title{
Classification of driver-assistance systems according to their impact on road safety and traffic efficiency
}

\author{
John Golias*, George Yannis and Constantinos Antoniou \\ Department of Transportation Planning and Engineering, National Technical University of \\ Athens, Athens GR-15773, Greece
}

(Received 15 February 2001; revised 11 July 2001; accepted 6 August 2001)

\begin{abstract}
The aim was to examine driver-assistance systems that seem to have a considerable potential for road safety and traffic efficiency improvement, and to propose an impact-oriented classification of these systems. A broad overview of a series of driver-assistance systems under development or in some cases already available is presented and it identifies the basic characteristics of each system and its expected impact on traffic efficiency and road safety. The latter is assessed on the basis of appropriate evaluation criteria. Expert judgement and literature evidence available are used in this context. This impact approach, in contrast with the usually adopted user or system-oriented approaches, allows for more appropriate identification of the priorities in the field of future research, development and promotion of driver-assistance systems. The proposed classification allocates the driver-assistance systems in four different categories on the basis of whether traffic efficiency and safety impact are high or low. This categorization reveals that $40 \%$ of the systems considered are expected to have a high safety and low traffic-efficiency impact, while only $15 \%$ is expected to have both impacts high.
\end{abstract}

\section{Introduction}

Today, the use of driver-assistance systems is of rapidly growing importance as these systems are expected to improve road safety, increase road capacity and attenuate the environmental impacts of traffic. The advent of new technologies supporting vehicle intelligence (sensors, transmitters, communications, computers) makes the use of driver-assistance systems less unapproachable to the wide public, allowing for safer and more efficient driver experiences.

Road safety is inherently associated with any new technology introduced in vehicles. The negative impacts of road accidents include fatalities, injuries, material damages and disruption of traffic, and the introduction of appropriate driverassistance systems could alleviate to a certain degree these consequences, both in terms of frequency and intensity (Sala and Mussone 2000). Driver-assistance systems have the potential to improve road safety by influencing traffic exposure, by reducing the probability of crashes and by reducing injury consequences. More precisely, driverassistance systems support the modification of the driving task by providing information, advice and assistance, they influence directly and indirectly the behaviour

* Author for correspondence; e-mail: igolias@central.ntua.gr 
of users of both equipped and non-equipped vehicles, and alleviate accident consequences by in-vehicle intelligent injury-reducing systems (Naniopoulos 2000).

Simultaneously, the deployment of driver-assistance systems is expected to influence traffic conditions. As such systems become increasingly popular and their penetration levels increase, the traffic dynamics are expected to change accordingly. These changes will be reflected to a variety of measures, including-but certainly not limited to-traffic capacity of links, mean driving speed and optimized headway distances. With the insightful use of driver-assistance systems, passenger car trips can be carried out in a more efficient way or shifted to more efficient modes of transport. This can be achieved, for example, through better real-time information for influencing pretrip choices, as well as offering in-vehicle information on passenger cars and on-trip information on public transport vehicles. Furthermore, better vehicle control and speed adaptation, as well as a more efficient fuel-saving driving style (by improved use of accelerator and gear shift), can be achieved by the use of driver-assistance systems.

The specific contribution of driver-assistance systems on road safety and traffic efficiency is something still under consideration and research. However, several attempts over the last decade (Brand et al. 1997) already revealed basic trends. Some systems present a net potential for road safety improvement, while some others have an effect mainly on traffic efficiency improvement. In other cases, improvement for road safety is often accompanied by lower traffic efficiency, whereas efficiency improvement can sometimes lead to the increase of the number of road accident. Furthermore, the level and rhythm of penetration of these systems as well as the implementation policy followed are determining factors for the impact of these systems to road safety and traffic efficiency.

Until today, several attempts concerning the classification of driver-assistance systems have been made. Very often, driver-assistance systems are classified according to the technologies (IT, wireless communications, etc.) and subsystems (autonomous in-vehicle, supported by GPS/GSM communication, linked with road infrastructure systems) used (EC 1997), or according to the vehicle type (passenger car, truck, bus) or type of road network (motorway, interurban, urban) they are referring to. When road safety features are examined, the distinct phases in the accident process (precrash, crash, post-crash) are often used for the classification of the driver-assistance systems (Heijer et al. 2000).

When functional analysis of the driver-assistance systems characteristics is attempted, these systems are initially classified according to the type of user (individual driver, professional driver, fleet owner, elderly drivers, etc.). Then these systems are classified according to the levels of driver tasks they are supporting, as strategic (route/mode choice, etc.), tactical (vehicle manoeuvring, etc.) and operational (steering, accelerator handling, etc.) (Michon 1985). For each level of driver tasks, driver-assistance systems can be further classified according to the driver subtasks they are referring to, such as perception (seeing, hearing, feeling, etc.), decision (for the various actions) and action (execution) (Sternberg 1969). Sometimes, driver-assistance systems are also classified according to the humanmachine interface they provide, like the provision of plain information, advices, warning messages, communication with the environment and to the capability of proceeding to a specific action.

The above classifications of driver-assistance systems reflect the current directions of research and development, which up to now concentrated either on 
the systems' improvement (technical advances) or on the driver behaviour identification (human-machine interface). Consequently, very often in the literature, driver-assistance classification follows either a system-oriented approach or a user-oriented approach, fully responding to the increasing complexity of driver assistance functions. However, these kinds of classifications cannot provide answers on the usefulness of driver-assistance systems, as the impact to traffic efficiency and road safety is not taken into consideration. Within this work, an impact-oriented approach for the classification of driver-assistance systems is attempted, where priorities for future developments can better be identified.

More precisely, a series of criteria for the identification of safety and efficiency impact are developed (Section 2), followed by the presentation and impact analysis of the various driver support systems (Section 3) and vehicle support systems (Section 4). The impact analysis was carried out mainly on the basis of expert judgement, supported in some cases by evidence in the literature. The outcome of this analysis allowed the formulation of an impact-oriented classification of driverassistance systems in Section 5. The overall conclusions are given in Section 6.

\section{Criteria for the identification of safety and efficiency impact}

The systems examined concerned either support of the driver or of the vehicle and are summarized in the following list.

Driver support systems:

- Driver information (navigation routing, integrated navigation, real-time traffic and traveller information).

- Driver perception (vision enhancement, electronic mirror, parking and reversing aid, state of the road surface systems).

- Driver convenience (driver identification, hands-free and remote control, automated transactions).

- Driver monitoring (driver vigilance monitoring, driver health monitoring).

Vehicle support systems:

- General vehicle control (automatic stop and go, platooning).

- Longitudinal and lateral control (speed control, adaptive cruise control, road and lane departure collision avoidance, lane change and merge collision avoidance).

- Collision avoidance (rear-end collision avoidance, obstacle and pedestrian detection, intersection collision warning).

- Vehicle monitoring (tachograph, alerting systems, vehicle diagnostics).

It is noted that, in practice, many of these driver-assistance systems are combined with each other, creating complex systems and serving more sophisticated functions. A good example is given by the platooning system, which integrates both lateral and longitudinal control of the vehicle. The combination possibilities are very large and it is not excluded that in the near future most of today's distinct driver-assistance systems will be integrated into one overall system.

The estimation of the impact of these driver-assistance systems to road safety and traffic efficiency was based on a number of preset criteria, specially selected for the purposes of this research. The criteria selected were those proposed in the 
international bibliography for measurements of road safety and traffic-efficiency impact, adapted for the purposes of this research. Only criteria mutually independent were selected allowing for a more straightforward impact analysis. For example, capacity increase was not considered as a traffic impact criterion, given that it can automatically be translated into improvement of mean speed. The criteria used are presented at the following list.

Criteria for estimating road safety impact:

- Avoidance of inappropriate speed.

- Keeping appropriate longitudinal and lateral distances.

- Support of driver awareness.

Criteria for estimating traffic-efficiency impact:

- Speed adjustment.

- Headway adjustment.

The criteria for estimating road safety impact correspond to road accident factors (Sabey and Taylor 1980), which are addressed by driver-assistance systems. The first criterion corresponds to the common problem of inappropriate speed for the specific traffic and road conditions, whereas keeping appropriate longitudinal and lateral distances with other vehicles and road elements refers to the difficulties of coordination within traffic conditions, especially complex ones. Finally, the support of driver awareness aims to deal with the driver fatigue and the necessary concentration on the driving tasks.

The criteria for estimating traffic-efficiency impact correspond to two basic traffic parameters, according to traffic engineering theory (Pline 1992). The adjustment (most often increase) of vehicle speed can result to a higher network efficiency either for the specific vehicle and/or for the complete network when several vehicles adjust their speed. Additionally, the adjustment (most often decrease) of vehicle headway can lead to more efficient traffic conditions as distances between vehicles are optimized. Speed and headway adjustment can both reflect the road capacity increase and the delays decrease.

In the following sections, the presentation of the systems examined adds a new dimension to the existing descriptive information, namely the analysis of the potential of each system for the improvement of road safety and traffic efficiency. The estimation of this impact was based on a combination of expert judgement and related findings from bibliography research and used the above impact analysis criteria. Expert judgement was necessary, as available quantified impact analysis results are very limited. For reasons of analysis organization not necessarily related to causal considerations the systems review follows a categorization in driver (Section 3) and vehicle support systems (Section 4).

\section{Driver support systems}

\subsection{Driver information}

The classic systems for driver information are those related to navigation routing, which provide location and route guidance input to the driver (Srinivasan and Jovanis 1997). Utilizing existing technology such as Radio Data System (RDS) 
and Traffic Message Channels, these systems combine static information from data sources such as compact discs or DVDs, with limited information pertaining to the traffic conditions along the vehicle route. Such systems often have the capability to perform simple routing operations and provide continuous driving directions, based on a number of simple criteria that the driver has preselected (e.g. minimum distance, minimum estimated travel time, minimum distance on highways, etc). Navigation routing systems can assist drivers in planning their route or finding their way in areas they are not particularly familiar with, leading thus in traffic efficiency and safety improvements related not only to the vehicle speed and longitudinal and lateral distances but also to the reduction in distance driven. Furthermore, the driver is expected to have an increased level of awareness, as the result of the system support. The equipped vehicle speed and headway with the preceding vehicle could also be slightly improved. Nevertheless, as traditional navigation systems do not incorporate real-time information, but instead are limited to using historical traffic information, they are not expected to provide directly significant safety or traffic efficiency benefits.

A number of integrated navigation systems have emerged in the past few years that support the driver through a variety of additional services such as signing, warning or even intervening in the driving process (e.g. by temporarily taking control of the vehicle), in the event of unsafe driving conditions, such as unsafe travel speed for the geometry ahead. Additional integration capabilities relate to the incorporation of environmental and road surface conditions (Hayward et al. 2000). These systems support the driver on his/her driving pattern by suggesting changes when unsafe driving behaviour is detected and consequently lead to safety benefits by the avoidance of inappropriate speed for the specific road and traffic conditions. No significant traffic-efficiency impact is anticipated from these systems as speed and headway benefits are not expected to be considerable.

Real-time traffic and traveller information systems combine the information available to users of traditional navigation systems with real-time travel-related information, which they receive from the infrastructure (e.g. through dedicated radio channels, roadside beacons or wide-area transmissions) (Spelt 1997, Wieck 1997). In general, the information that the user receives can be either descriptive (Kantowitz et al. 1997) or prescriptive (guidance) (Bonsall and Parry 1991). Descriptive systems broadcast current or future traffic conditions, as well as information on incidents or other relevant information to assist drivers in the determination of their routes. Some systems provide the user with a set of available routes and additional information (e.g. travel times for each route) to assist the driver to select the optimal route. A limited number of systems have the ability to let the user specify criteria for the determination of the best route (e.g. fastest route, route that avoids interstates, route that avoids toll roads). Another category of systems generally referred to by the term prescriptive, determine the best path and broadcast it to the user. Real-time traffic and traveller information systems can exploit information such as vehicle location, previous route guidance instructions, safety and advisory information, and other real-time updates on conditions such as congestion, work zones, environmental and road surface conditions (Brand et al. 1997, Davison et al. 1997). Unlike traditional navigation systems, real-time information systems consider prevailing traffic conditions, thus providing a means of overcoming unexpected traffic irregularities. Furthermore, such systems can exploit information on planned events (such as sports games or scheduled road maintenance work). Therefore, the safety 
benefits from the introduction of such systems in the speed, distance and driver awareness are not expected to be measurable, but the speed increase of the equipped vehicles could result in significant traffic efficiency gains. Possible reduction in distance driven is expected to have a positive impact on both traffic efficiency and safety. Nevertheless, significant headway improvements are not anticipated, as realtime traffic and traveller information systems do not assist vehicle control.

It is noted that negative safety consequences from the above driver information systems are possible, as they require in-vehicle screens, which may distract the driver's attention from his primary driving task. Research on driver behaviour towards the use of these systems, especially during the period of the introduction of the new systems is considered necessary not only for the identification of the overall safety impact but also for the appropriate redesign of the systems, if necessary.

\subsection{Driver perception}

Vision enhancement systems can augment the driver's vision under conditions of reduced visibility, e.g. due to fog, rain, snow or darkness. Such systems require invehicle equipment to sense, process and display the information, ranging from specially designed headlights to infrared and radar sensors (Mahach et al. 1997). Data collected from in-vehicle systems can be combined with information obtained from road sensors and transmitted wirelessly to the vehicle to sense the danger of imminent collisions. The information is gathered in an on-board computer that processes it and compares it to preprogrammed safety limits. Generated collision warning signals can then be displayed on head-up displays (HUD) or transmitted as audible signals through the vehicle audio system to the driver (Chassant 2000). Vision enhancement systems may have positive impacts in the maintaining of safe longitudinal and lateral distances by the driver. As drivers are more aware of the traffic dynamics of the vehicles around them (both in their direction, as well as the opposite direction), then they can maintain safer distances from these vehicles. In terms of speed and driver awareness, vision enhancement is not expected to result into identifiable safety benefits. Furthermore, as these systems improve the visibility ahead they are expected to have significant impact on the speed of the equipped vehicles, as the drivers will be more comfortable driving in higher speeds. On the contrary, vision enhancement systems are not expected to have significant impacts on the traffic efficiency through headway reduction.

Rear-view mirrors have been core safety equipment in cars for several decades. Nevertheless, limitations inherent in the materials used, as well as the structural elements of the vehicles, result in poor viewing conditions and the existence of blind spots that often lead to accidents. Video cameras and radar/infrared/thermal sensors can substitute traditional rear-view mirrors and display an image of the entire rear view of the vehicle in a special screen in the dashboard. Such systems are collectively referred to as electronic mirrors (EADM 1999). As watching a screen can distract the drivers from their primary driving tasks, several systems can process the electronic mirror images and warn the driver - using an audio or visual alarm - in the case of a dangerous situation, e.g. when the distance between vehicles in the same or adjacent lanes has dropped below a predetermined safety threshold. Such electronic mirror systems assist the driver in maintaining safe distances with vehicles in the same or adjacent lane and are particularly helpful in dealing with vehicles that are behind the equipped vehicle, e.g. during lane change manoeuvres. As these systems focus primarily in the traffic behind the equipped vehicle, they are not expected to have a 
great positive impact on the vehicle speed or headway. The automatic driver warning capability in the event of unsafe driving environment increases the awareness of the driver and thus may lead to additional safety gains.

Manoeuvres involving reverse operations present additional difficulty to most drivers. Parking and reversing aid systems provide continuous information and early warning to drivers performing such operations. To accomplish this task these systems use various types of sensors to achieve short-range obstacle detection and tracking. Parking and reversing aid systems assist the driver's perception of areas that are out of sight during the execution of delicate manoeuvres and warn the driver of potentially unsafe close distance to obstacles, offering a reasonable time-window for the driver to react accordingly and avoid collision. The traffic or safety impacts of such systems are not particularly evident, but similar systems may be popular as they absorb part of the inconvenience associated with parking and reverse manoeuvres. It is obvious, that as these systems do not apply to the majority of the driving tasks, their impact on safety and traffic efficiency may not be traceable.

A promising category of driver information systems refers to systems collecting and analysing information on the road surface conditions using vehicle-mounted or fixed infrastructure road sensors (Cremona et al. 1994). The collected information can then be transmitted to the in-vehicle system, where it is further processed. If unsafe road surface conditions are identified ahead of the vehicle, then the driver can be notified via audio or visual message. Furthermore, the information can be transmitted to the appropriate traffic information centres, from where it can be disseminated to other road users as well as the appropriate authorities. The impacts of such systems could be significant as drivers' awareness of the road surface conditions can have considerable positive impacts on both safety (as a combination of appropriate driving speed adoption, appropriate headway adoption, and increased driver awareness) as well as traffic efficiency, through better driving behaviour in relation to speed.

\subsection{Driver convenience}

Driver convenience is a key factor determining their performance. Systems already commercially available in high-end luxury cars offer the capability to identify the driver (from a choice set of a few preconfigured drivers) and automatically adjust the seat, the steering wheel, the rear and side mirrors, the temperature, etc. to the particular driver's preset preferences. The impact of driver identification systems on driver awareness can be significant, as the driver is not distracted by trying to adjust the driving environment (seat, steering wheel, rear and side mirrors). Furthermore, the driving environment always adjusts to the optimal settings for each driver, whereas manual reconfiguration-often demanding several complex or timeconsuming activities - could be bypassed, e.g. during short trips, thus compromising driver's performance and safety. As these systems do not affect driving decisions directly, they are not expected to produce direct safety or traffic efficiency benefits due to speed and/or headway improvements.

Electronic appliances, such as cell phones, fax machines, personal computers, etc. find their way into passenger cars. Operation of such systems invariably distracts the driver, substantially limiting the driver's capability to respond properly to emergency situations before an accident. Various systems have been developed to overcome this issue, including hands-free interfaces and remote control units located on the steering wheel (Hofmeister et al. 1997). While the 
former offer the highest safety advantages, the latter are often a significant step towards the right direction for situations where control can not be achieved through a hands-free interface; at least the driver's hands do not depart from the steering wheel. Such systems may have positive impacts on driver's awareness, as potential distractions from the non-driving-related information sources are minimized. A potential safety pitfall, however, is that the drivers of equipped vehicles may be more inclined to use such systems, on the assumption that they do not interfere with the driving performance; even though hands-free systems may alleviate the distractions of the users from their driving-related tasks, it is clear that any non-related task impedes the driving performance. As the systems belonging in this category are not directly involved in the driving process, they are not expected to significantly affect road safety and traffic efficiency.

Automated transaction systems facilitate high-speed electronic transactions, often without even the need for the vehicle to slow or stop. Examples of such systems include electronic toll collection, parking fee payment, and parking/ restricted area entry permit verification (Venable et al. 1995, Abe et al. 1996). Some of the key technologies that have made such systems possible are smartcards (Blythe 1997), GSM and dedicated short-range communications (DSRC) (CEN 2000). As these systems have substantial infrastructure requirements and serve a number of vehicles, standardization is required that will ensure the interoperability of such systems (Hamet 1999). The positive safety impact of automated transaction systems is related to the elimination of unnecessary and sometimes dangerous deceleration and acceleration areas. As this is an indirect effect, its magnitude is not expected to be considerable. Automated transaction systems improve traffic efficiency by making transactions such as tolling transparent to the traffic; instead of having to stop to pay the toll fare, vehicles can drive through the control station, often at speeds up to $180 \mathrm{~km} / \mathrm{h}$. Furthermore, as the vehicles are not obliged to decelerate and accelerate (as they would have to do in the case of a non-automated transaction), headwayrelated traffic efficiency gains are expected as well.

\subsection{Driver monitoring}

Driver vigilance monitoring systems continuously monitor the driver's performance for possible signs of conditions that may endanger the driver, such as drowsiness as a consequence of fatigue (Sugasawa et al. 1996), alcohol abuse, medication, etc., or lack of attention, e.g. due to stress (Grace 1999, Boverie et al. 2000). When the system detects potential problems with the driver's awareness then the system uses audio and visual warnings to draw the driver's attention and restore an acceptable level of alertness. Such systems are expected to have high impact in maintaining driver's awareness especially during long interurban trips and can consequently lead to considerable safety benefits.

Driver health monitoring systems take driver monitoring a step further by monitoring several parameters of the driver's health conditions and combining the results to estimate the current health level of the driver. From this information, the system assesses the condition of the driver and if it appears to be below certain preselected 'safe' levels then the driver and possibly some external entity, e.g. a doctor or the police, are notified (Hernandez et al. 1998). Obviously, such systems could have significant awareness-related safety impact as the driver is notified when his health is deteriorating. 


\section{Vehicle support systems}

\subsection{General vehicle control}

Automatic stop-and-go systems allow vehicles to automatically stop when this is necessary, e.g. the preceding vehicle has stopped, and start again when the conditions allow it (Carrea 1993). Such systems could offer significant safety benefits in hazardous situations and in situations where frequent stop-and-go is required, such as congested conditions. A key issue with automatic stop-and-go systems that are being developed has been a relatively high false alarm rate; overcoming this problem could lead to a wider use of such systems (Brand et al. 1997). Automatic stop-and-go system s could have significant positive safety impacts by managing the vehicle speed and longitudinal distance better than the human driver. Nevertheless, these systems generally would not improve the driver's awareness, especially as the driver could increasingly rely more on the system's performance. Finally, as stop-and-go usually applies to congested conditions limited significant traffic efficiency gains are expected, mainly through improved headway maintenance.

Another system in this category, albeit with a lower level of maturity is platooning, a situation where each vehicle travels keeping a constant headway from the preceding one, either through external speed control or through electronic speed control by the vehicle itself. A special case of this function is the tow-bar application, where the vehicles (usually trucks) are electronically coupled and each follows the preceding one. Platooning application areas are usually restricted to highway and motorway network sections with a reduced speed limit (usually up to $85 \mathrm{~km} / \mathrm{h}$ ). The more advanced platooning systems, where the vehicles are externally controlled by the infrastructure, are called intelligent vehicle highway systems (IVHS) (Brand et al. 1997). Platooning systems could offer safety benefits as the vehicle's speed and position are electronically controlled. Platooning systems widespread use could result in significant traffic efficiency benefits as vehicles - that have formed platoons achieve higher speeds and shorter headways.

The most well-known longitudinal control system is probably the variable speed limiter or the intelligent speed adaptation. Different implementations range from external speed recommendations to an automatic speed reduction function. The latter may be imposed directly to all (equipped) vehicles within the control area, e.g. through a communication centre, or indirectly, e.g. by managing the local traffic lights accordingly. Stop and go functions may be also included in this category, especially when implemented in conjunction with an infrastructure-based system (Davison et al. 1997, Oei 1998b). Speed-limiting systems may provide significant safety improvements as they offer a way to effectively control speed and longitudinal distance between cars. The safety impacts of such systems through improvement of driver's awareness are not expected to be high. Obviously, such speed-limiting systems could not have considerable positive impacts on traffic efficiency.

Adaptive cruise control (ACC) is a more elaborate longitudinal control system which adjusts vehicle speed to maintain a safe separation with the preceding vehicle (Martin 1993, Winner et al. 1996). Adaptive cruise control senses the presence and relative velocity of moving vehicles ahead of the equipped vehicle and adjusts the speed of travel accordingly (Oei 1998a, Hayward et al. 2000). Adaptive cruise control systems could have significant positive safety impacts as they effectively control the speed and longitudinal distance between vehicles, ensuring that no rear-end collisions occur. However, driver's awareness is not influenced in a way that could 
lead to significant safety benefits. Furthermore, as the speed and headway are automatically control they are optimized and can therefore provide better traffic performance compared with human control.

It is noted that negative safety consequences from the introduction of these general vehicle control systems may be encountered, as the driver could be surprised in his driving task by the automatic actions undertaken by the systems. Furthermore, depending on the settings of the various vehicle control systems (maximum speed and headway) negative impacts on traffic may appear. Only further driver behaviour research and appropriate redesign of the systems will allow the minimization of any negative impact due to these systems.

\subsection{Collision avoidance}

Road and lane departure collision avoidance is a lateral control system providing warning and control assistance to the driver through lane or road edge tracking and by determining the safe speed for road geometry in front of the vehicle (Pomerleau et al. 1997). The system continuously calculates the vehicle's optimal position and compares it with the actual vehicle position. If the system detects deviations exceeding the defined safety thresholds, then it creates audiovisual warnings for the driver. More advanced systems could feature extended functionality, including particular suggestions/directions to the driver on how the particular problem can be overcome or actual control interventions to restore the vehicle's intended path. Such systems may have a positive impact on safety by ensuring that vehicles do not inadvertently depart from their desired lane, a situation that can often result in an accident. The safety impacts of these systems on speed and driver awareness are not expected to be significant. Similarly, as these systems are only activated in the event of an emergency situation (road or lane departure) the direct impact on the traffic efficiency is not expected to be traceable.

Lane change and merge collision avoidance systems are another type of lateral control systems providing various levels of support for detecting and warning the driver of vehicles and objects in adjacent lanes. While this information can be useful during normal driving conditions, it is particular valuable during lane change or merge manoeuvres. Systems in this category track vehicles in adjacent lanes and use this information to warn the driver when their position and/or speed makes the planned lane change/merge manoeuvre unsafe. More sophisticated systems may include speed and steering control intervention for enhanced collision avoidance. Such systems ensure that lateral separation between vehicles in adjacent lanes are always maintained and may therefore have significant positive impacts in the reduction of traffic accidents (Mazzae et al. 1995, Young 1995, Campbell et al. 1996). The optimized lane change and merging capabilities of these systems may lead in significant traffic efficiency gains, related to better headways. These systems are expected to have little or no impact on the vehicle's speed.

Rear-end collision avoidance systems sense the presence and speed of vehicles and objects in the vehicle's lane of travel and provide to minimize the risk of collisions with vehicles and objects found in front of the equipped vehicle (Ganci et al. 1995, Woll 1995). More sophisticated versions can include longitudinal control through vehicle braking and speed adaptation, and ultimately even lateral control by offering lane change capabilities to avoid collisions. Rear-end collision avoidance systems could offer safety improvements by monitoring the lane in which the vehicle is travelling for slow moving 
vehicles and other obstacles and adjusting the equipped vehicle's speed and travelling lane accordingly to avoid a collision. It is obvious that systems that adjust the vehicle's speed-such as rear-end collision avoidance-can not have significant safety or traffic efficiency benefits associated with optimized speed. In addition, the impact of such systems in driver awareness is not expected to produce enough potential for safety benefits.

Obstacle and pedestrian detection systems offer similar services by warning the driver when pedestrians, vehicles, or obstacles are in close proximity to the driver's intended path (Butsuen et al. 1996, Kamiya et al. 1996, Sugasawa et al. 1996, Papageorgiou et al. 1998). Information from on-board sensors or infrastructurebased sensors is used to detect obstacles and pedestrians and speed and direction information from the on-board computer is used to estimate the vehicle's path. The combination of the above information may generate alarms notifying the driver of potentially unsafe conditions. Even though the operation of such systems is similar to that of the rear-end collision avoidance systems, obstacle and pedestrian detection systems offer different safety benefits. In particular, as obstacle and pedestrian detection systems provide warnings to the driver when potential collision danger is imminent, positive safety impacts related to vehicle speed and driver awareness may be achieved. Furthermore, as these systems may only limit the vehicle speed (as a result of obstacle and pedestrian warnings), no significant traffic efficiency benefits are expected.

Most accidents happen at intersections. Intersection collision warning systems utilize a cooperation of vehicle and infrastructure systems to provide warning to the driver for potential collision at an intersection (Lloyd et al. 1996, Brand et al. 1997). A special category of intersection collision avoidance systems is railroad crossing collision avoidance systems, which provide in-vehicle warnings to drivers approaching railroad crossings when a train is approaching (Luedeke 1997, Polk 1997). Initial implementation of this feature is anticipated for buses and trucks carrying hazardous cargo. Extensions to other vehicles may become feasible when this technology becomes more cost-effective. One way to achieve this is by creating economies of scope, i.e. combine the system with other services. Intersection collision warning systems may provide considerable safety benefits by limiting on time the speed of the equipped vehicle, when collision danger is sensed at a downstream intersection. Furthermore, such systems may improve the driver's awareness by notifying, e.g. by audio or visual messages, the driver about potentially dangerous conditions that the system has identified. Finally, these systems are not expected to affect headway and speed adjustment in a way that could influence traffic efficiency.

\subsection{Vehicle status monitoring}

A simple system for vehicle status monitoring is the tachograph recording equipment which can record, store, display, print, and output data related to driver activity, as well as log information describing the beginning and end of each trip, control activities performed during the trip, e.g. by the police, etc. Tachograph system s can also log additional information allowing for more sophisticated analysis when the tachograph information is analysed, e.g. the ability to detect operating violations, such as driving for a period exceeding the maximum designated period without a stop or exceeding the speed limit for the specific type of vehicle. The existence of a tachograph in the vehicle may force the driver to be more alert, drive at safer speeds and maintain optimal distances from other vehicles, thus improving 
safety. It is, however, obvious that the contribution of such auditing equipment in the improvement of traffic efficiency is not expected to be considerable.

A large number of alerting systems have been developed aimed at alerting the emergency services (e.g. police, ambulance, fire brigades, highway patrols) in case of a traffic incident. Furthermore, some of these systems offer also dedicated support services, to which the troubled drivers get connected automatically. Most of the systems feature either a cell-phone technology connection or satellite-based communications (Benson and Clima 1996, Heddebaut and Rioult 1998). Alerting systems can either be absolutely automated and/or require a-more or lesssubstantial intervention from the driver of the vehicle in emergency (Sobolewski and Deeter 1997). For example, automatic collision notification system calls automatically for emergency services when the vehicle is involved in a serious crash and it also supports manual operations to call for emergency or other types or roadside assistance. The vehicle location is automatically transmitted with the call for assistance in either the automatic or manual mode of operation. Position accuracy will be sufficient to determine direction of travel on a divided highway. Beyond the notification/alert services, these systems take advantage of the existing infrastructure to bundle a number of other services, including stolen vehicle tracking, remote door unlock, roadside assistance in case of car breakdown, route support upon request, remote diagnostics of vehicle malfunctions and driver condition. Alerting systems do not intervene with the driving tasks and therefore are not expected to yield significant safety or traffic-related benefits.

Vehicle diagnostic information systems are an extension of current vehicle monitoring and self-diagnostic capabilities, offering elaborate engine condition information services such as oil pressure and coolant temperature gauges (Bannatyne and Warshawsky 1997). Such systems monitor vehicle safety-related functions, compare the readings with the expected operating conditions and warn the driver or the operator if potentially irregular operation is detected. Diagnostic information services can be considered as an added diagnostic capability for existing functions like monitoring braking system integrity, sensor and actuator performance, and the communication system. Like the alerting systems, vehicle diagnostic systems do not intervene with the driving tasks and therefore no significant safety or traffic-related benefits are expected.

\section{Impact-oriented classification of driver-assistance systems}

On the basis of the above presentation and impact analysis of driverassistance systems, a high or low impact value was assigned to each of the preselected criteria (see Section 2) for each driver-assistance system examined. In this way, table 1 was prepared, which clarifies the degree of safety and efficiency impact of the systems considered.

It is noted that the estimation of the impact of driver-assistance systems refers only to direct impact on road safety and traffic efficiency. Certainly, there is often indirect impact, as for example the avoidance of an accident (i.e. the decrease in the number of road accidents) may lead to the avoidance of a related congested situation (i.e. less congestion). However, for the purposes of this research, only direct impact was considered in the analysis of each system.

It is noted that in some cases, driver-assistance systems with significant quantities of information on in-vehicle screens (e.g. navigation routing, electronic mirrors, etc.) may distract the driver's attention and thus they may have negative consequences on 
Classification of driver-assistance systems

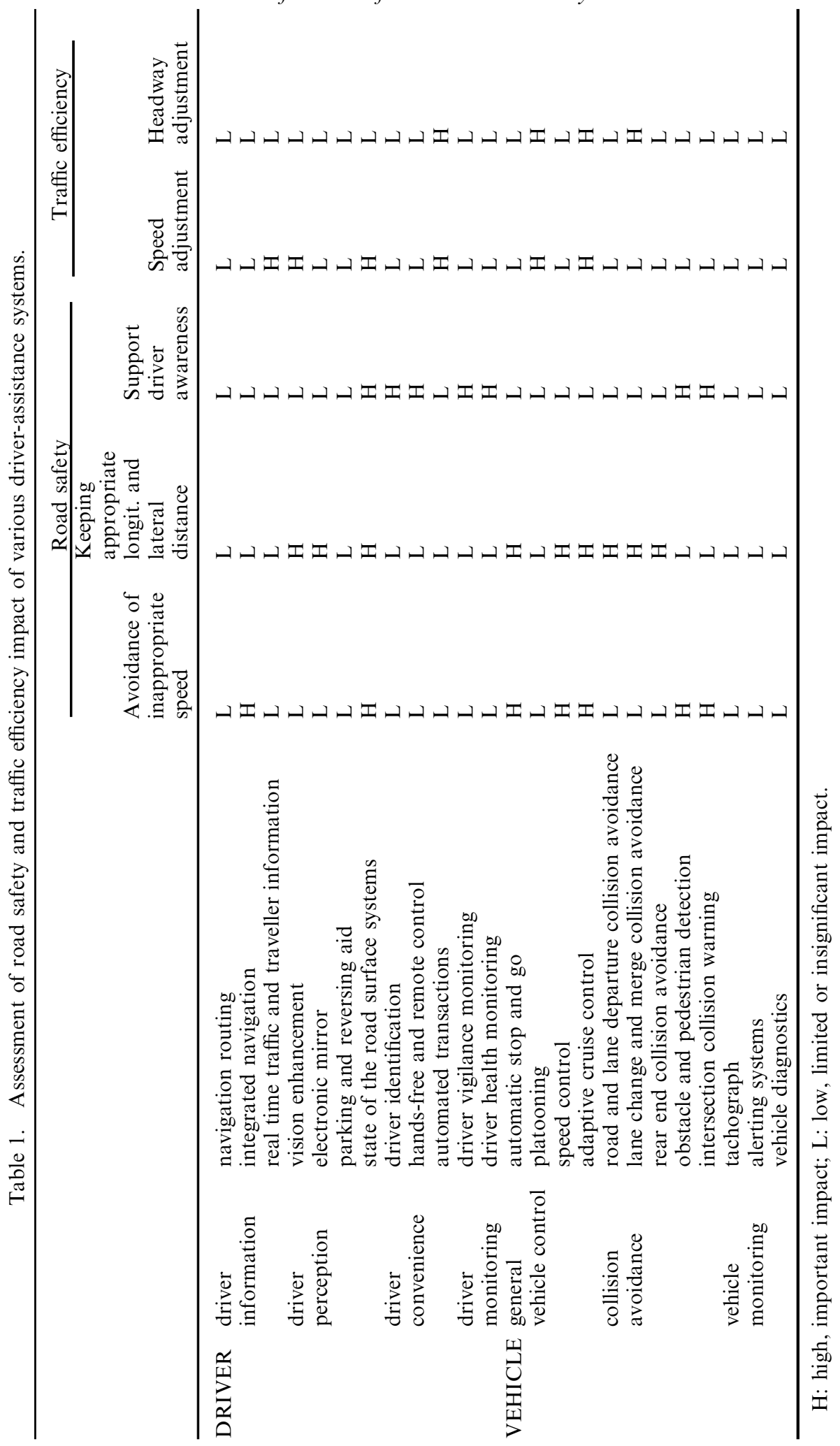


Table 2. Impact-oriented classification of driver-assistance systems.

\begin{tabular}{|c|c|c|c|}
\hline \multirow{20}{*}{$\begin{array}{l}\text { Traffic } \\
\text { efficiency } \\
\text { impact }\end{array}$} & \multirow{6}{*}{ High } & \multicolumn{2}{|c|}{ Road safety impact } \\
\hline & & High & Low \\
\hline & & state of the road surface systems & automated transactions \\
\hline & & adaptive cruise control & platooning \\
\hline & & lane change and merge & real time traffic and traveller \\
\hline & & collision avoidance & information \\
\hline & & vision enhancement & \\
\hline & Low & automatic stop and go & navigation routing \\
\hline & & speed control & parking and reversing aid \\
\hline & & obstacle and pedestrian detection & tachograph \\
\hline & & intersection collision warning & alerting systems \\
\hline & & integrated navigation & vehicle diagnostics \\
\hline & & electronic mirror & \\
\hline & & driver identification & \\
\hline & & hands-free and remote control & \\
\hline & & driver vigilance monitoring & \\
\hline & & driver health monitoring & \\
\hline & & road and lane departure & \\
\hline & & collision avoidance & \\
\hline & & rear end collision avoidance & \\
\hline
\end{tabular}

the safety level. In the framework of this research, it is assumed that any negative impacts are limited. However, further research is required towards determining the actual driver behaviour due to in-vehicle screens, which will clarify the overall net balance of these systems in relation to safety.

The above impact analysis of driver-assistance systems led to the formulation of an impact-oriented approach for the classification of the driver-assistance systems, as shown in table 2. Here, a system is put in a category of high impact only if at least one of the criteria has a value of high impact. Within each part of table 2, the systems are put in order of importance, with those having the more values of high impact at the top.

It is interesting to note from table 2 that only four driver-assistance systems present high values for both road safety and traffic-efficiency impact (state of the road surface systems, adaptive cruise control, lane change and merge collision avoidance and vision enhancement), with the first two systems presenting highimpact value for four out of five criteria. Twelve other systems present a high road safety impact but limited traffic-efficiency impact, whereas only three systems present important high traffic-efficiency impact and limited safety impact. Finally, another five systems present limited impact to both road safety and traffic efficiency. The fact that there are more systems with important impact on road safety (16 systems) than systems with important impact on traffic efficiency (seven systems) was expected, as the primary objective for most of the driver-assistance systems is the improvement of road safety.

\section{Conclusions}

Today, the current information technology applications together with several developments in the car-industry allow for fast and efficient communication 
networks, real-time processing of large amount of data and user friendly humanmachine interfaces (Rumar et al. 1999), which make possible the reliable and costeffective use of several driver-assistance systems. In this way, driver-assistance systems are incorporated in the new vehicles, responding better to the diverse needs of the road users for safer and more efficient traffic conditions. Subsequently, the existence of strong evidence that the impact of driver-assistance systems to road safety and traffic efficiency is positive, will progressively make the use of these systems much more attractive, as their fixed and operational costs are steadily decreasing.

The comprehensive and critical review of several existing driver-assistance systems carried out in the framework of this work, allowed for the macroscopic identification of the impact of these systems to road safety and traffic efficiency. The output of this critical review led to the formulation of an alternative classification approach of these systems based on the systems' impact to road safety and traffic efficiency, escaping from the traditional classifications, which follow system or user oriented approaches. As driver-assistance systems reach their maturity, the proposed system performance based classification is more suitable for their further development, coupling the existing work on systems availability with work on systems usefulness. Besides, the appropriate development process of any type of system should be based on the suitable balance between system availability, attempting to provide answers to basic questions concerning issues like whether the systems required are produced and whether the systems produced are required.

The proposed classification proposes a ranking of driver-assistance systems, where at the top are found the systems presenting high values in the preselected road safety and traffic-efficiency impact criteria. The systems providing real-time information for the road surface systems as well as those related to adaptive cruise control are ranked at the top impact levels followed by systems related to lane change and merge collision avoidance as well as vision enhancement. From the proposed classification it appears that more systems present high safety impact than high-efficiency impact, a result possibly attributed to the fact that historically driverassistance systems were primarily aiming at road safety improvement .

This impact-oriented classification allows for another view of the particularities of driver-assistance systems, leading thus to the identification not only of the areas where future research is needed, but also of the priorities for further system development and promotion. It is obvious that development and application of driver-assistance systems should be carefully monitored and not left only to industry and market forces, especially when road safety issues are addressed. Additionally, this classification should allow for the identification of the necessary legislation actions for mandatory standards or procedures at international level to ensure the proper design of new intelligent transportation systems.

The results of this research make obvious the need for further experimental and laboratory research in the field of quantifying road safety and trafficefficiency impact of driver-assistance systems. When more quantified and modelbased impact analysis (at both micro and macro scale) is available, the proposed systems classification can be more detailed and consequently more useful for the better identification of the priorities for the development of driver-assistance systems. Certainly, impact analysis should not be limited to microscale modelling but also to network and transportation system modelling as well as to 
multicriteria analysis allowing for the identification of the overall system impact to safety, efficiency, environment and the economy.

Finally, this critical review of driver-assistance systems revealed that the systems performance and impact is related to a number of developments concerning to the concept of intelligent road, the human-machine interface and the systems implementation transition phases. Consequently, related further research in these fields should be coupled with the corresponding research on the driver-assistance systems so that an efficient and operative outcome is achieved.

\section{References}

Aвe, A., Hori, T. and Ofuchi, K., 1996, Observations of the effect on drivers of lane guidance function employing in-vehicle voice guidance and display units [CD-ROM]. In Proceedings of the Third Annual World Congress on Intelligent Transport Systems, Orlando, FL.

Bannatyne, R. and Warshawsky, E., 1997, The changing world of automotive electronic control units. In Proceedings of the Future Transportation Technology Conference (Warrendale: Society of Automotive Engineers), paper 972638.

Benson, J. L. and Clima, B. T., 1996, Implementing a MAYDAY system: institutional issues and protocol involved in implementing vehicle MAYDAY technologies. In Third World Congress on Intelligent Transport Systems, Orlando, FL.

Blythe, P. T., 1997, Implementing smart card technology to transform transport payment and customer information. In Proceedings of the 4th World Congress on Intelligent Transport Systems (Berlin: ICC).

Bonsall, P. and Parry, T., 1991, Using an interactive route-choice simulator to investigate drivers' compliance with route guidance advice. Transportation Research Record, 1306, $59-68$.

Boverie, S., Devy, M., Le Quellec, JM., Mengel, P. and Zittlau, D., 2000, 3D perception for vehicle inner space monitoring. In AMAA Conference, Berlin, Germany, March.

Brand, C., Davison, P., Lewis, A., Moon, D., Site, P. D., Gentile, C., Filippi, F., Landolfi, O., Dougherty, M., Korver, W., Harrell, L., van Toorenburg, J., Akerman, J., Eriksson, E. A., Hernandez, H., Weber, M., Daunerv, A., Heckelsmueller, J., Hoffmann, A., Leiss, U., Linde, E. and Petzel, W., 1997, Forecast of New Technologies with Major Impacts. EU project FANTASIE, deliverable 9 November.

Butsuen, T., Yoshioka, T. and Окuda, K., 1996, Introduction of the Mazda advanced safety vehicle. In Proceedings of the 1996 IEEE Intelligent Vehicles Symposium, Tokyo, pp. $242-247$.

Campbell, J. L., Hooey, B. L., Carney, C., Hanowski, R. J., Gore, B. F., Kantowitz, B. H. and Mitchell, E., 1996, Investigation of Alternative Displays for Side Collision Avoidance Systems (Final Report) (Seattle: Battelle Research Center).

CArrea, P., 1993, Integration between anticollision and AICC functions: the Alert Project. In Proceedings of the Intelligent Vehicles '93 Symposium (Tokyo: IEEE).

CEN TC 278 WG 9, 2000, Dedicated Short-Range Communications in close cooperation with ISO TC 204 WG 15 'DSRC for TICS Applications', Road Transport and Traffic Telematics - Dedicated Short-Range Communications.

Chassant, Y., 2000, La vision de nuit. Auto Concept Magazine, March/April.

Cremona, P., Kunert, M. and Castinie, F., 1994, Parametric spectrum analysis for target characterization. In Proceedings of the Intelligent Vehicles '94 Symposium (Paris: IEEE).

Davison, P., Brand, C., Lewis, A., Moon, D., Site, P. D., Gentile, C., Filippi, F., Landolfi, O., Dougherty, M., Korver, W., Harrell, L., van Toorenburg, J., Akerman, J., Dauner, A., Heckelsmueller, J., Leiss, U., Linde, E. and Petzel, E., 1997, A Structured State-ofthe-Art Survey and Review. EU project FANTASIE, deliverable 8 November 1997.

EADM, 1999, Electronic mirror merges views. European Automotive Design Magazine, June. European Commission, 1997, Community Strategy and Framework for the Deployment of Road Transport Telematics in Europe. COM (7) 223 final (Brussels: EC).

Ganci, P., Pотts, S. and Okurowski, F., 1995, Forward-looking automotive radar sensor. In Proceedings/SPIE - The International Society for Optical Engineering, 2592, pp. 60-65. 
Grace, R., 1999, A drowsy driver detection system for heavy vehicle. Paper presented to the Conference on Ocular Measures of Driver alertness, Herndon, VA.

Hamet, P., 1999, Europe-The General Direction. TOLLtrans Supplement to Traffic Technology International, October/November, 10-11.

Hayward, M., Becker, S., Nilsson, L., Brockmann, M. and Sala, G, 2000, TR1004, ACASSIST, Anti-Collision Autonomous Support and Safety Intervention System (incorporating ROADSTER), submitted as project deliverable D3. 1: Report on Users' Needs.

Heddebaut, M. and Rioult, J, 1998, Appel d'urgence, localisation d'usagers en detresse et alerte rapide des conducteurs. Analyse technique des solutions en cours de déploiement. TEC journal, 150, $11-19$.

Heijer, T., Oei, H. L., Wiethoff, M., Boverie, S., Penttinen, M., Schirokoff, A., Kulmala, R., Heinrich, J., Ernst, A. C., Sneek, N., Heeren, H., Stevens, A., Beriaris, A. and Damiani, S., 2000, Problem identification, user needs and inventory of ADAS, ADVISORS project deliverable D1/2.1.

Hernandez-Gress, N., Estève, D. and Bekiaris, A., 1998, IMU: integrated monitoring unit of the SAVE diagnostic system. In Intelligent Component for Vehicles Workshop (ICV '98), Seville, Spain.

Hofmeister, A., Mattos, P., Nicolai, J. and Kasparian, M., 1997, The Driver Information System for the Rest of Us. Paper 970171 (Warrendale: Society of Automotive Engineers).

Kamiya, H., Fujita, Y., Tsurga, T., Nakumra, Y., Matsuda, S. and Enomoto, K., 1996, Intelligent technologies of Honda ASV. In Proceedings of the 1996 IEEE Intelligent Vehicles Symposium, Tokyo, pp. 236-241.

Kantowitz, B. H., Hanowski, R. J. and Kantowitz, S. C., 1997, Driver acceptance of unreliable traffic information in familiar and unfamiliar settings. Human Factors, 39, $164-176$.

Lloyd, M. M., Bittner, JR, A. C. and Pierowicz, J. A., 1996, Driver-vehicle interface (DVI) design issues of an intersection collision avoidance (ICA) system [CD-ROM]. In Proceedings of the Third Annual World Congress on Intelligent Transport Systems, Orlando, FL.

Luedeke, J., 1997, Highway Railroad Grade-Crossing Warning Device and Barrier System Technologies for High-Speed Applications, vol. III (Draft Report) (Columbus: Battelle Memorial Institute).

Mahach, K., Knoblauch, R. and Simmons, C., 1997, A Preliminary Field Evaluation Test of Ultraviolet-Activated Fluorescent Roadway Delineation (FHWA-RD-97-082) (Washington, DC: FHWA).

Martin, P., 1993, Autonomous Intelligent Cruise Control Incorporating Automatic Braking. Technical Paper 930510 (Warrendale: Society of Automotive Engineers).

Mazzae, E. N., Garrott, W. R. and Flick, M. A., 1995, Human Factors Evaluation of Existing Side Collision Avoidance System Driver Interfaces. Technical Paper 952659 (Warrendale: Society of Automotive Engineers).

Michon, J., 1985, A critical view of driver behavior models: what do we know, what should we do? In L. Evans and R. C. Schwing (eds), Human Behavior and Traffic Safety (New York: Plenum), pp. 485-524.

Naniopoulos, A., 2000, Advanced driver-assistance systems and traffic safety. In Proceedings of Workshop on 'The role of Advanced Driver Assistance Systems on traffic safety and efficiency', organized by NTUA and AUTh, Athens, 18 October.

Oei Hway-liem, 1998a, Advanced Cruise Control (ACC): A Literature Study (Leidschendam: SWOV). [in Dutch]

Oei Hway-Liem, 1998b, Intelligent Speed Adaptation (ISA). A Literature Study (Leidschendam: SWOV). [in Dutch]

Papageorgiou, C., Evgeniou, Th. and Poggio, T, 1998, A trainable pedestrian detection system. MIT, CBCL and AI Laboratory Cambridge, USA. In 1998 IEEE International Conference on Intelligent Vehicles.

Pline J., 1992, Traffic Engineering Handbook, 4th edn (Englewood Cliffs: Prentice Hall for the Institute of Transportation Engineers). 
Polk, A. E., 1997, ITS applications to railroad crossing safety: a summary of U.S. activities [CD-ROM]. In Proceedings of the ITS America Seventh Annual Meeting (Washington, DC: ITS America).

Pomerleau, D., Thorpe, C. and Emery, L., 1997, Performance specification development for roadway departure collision avoidance systems. In Proceedings of the 4th World Congress on Intelligent Transport Systems (Berlin: ICC).

Rumar, K, Carsten, O., Fleyry, D., Heijer, T., Kildebogaard, J., Kulmala, R., Lind, G., Machata, K., Mauro, V., Zackor, H., Berry, J., Breen, J. and Ward, M., 1999, Intelligent Transportation Systems and Road Safety (Brussels: European Transport Safety Council).

SAbey, B. E. and TAYlor, H., 1980, The Known Risks We Run: The Highway. Report SR 567 (Crowthorne: TRRL).

Sala, G. and Mussone, L., 2000, The potential impact on traffic safety of lateral support systems. In Proceedings of the 7th World Congress on ITS, Turin, Italy.

Sobolewski, M. and Deeter, D., 1997, Multi-Jurisdictional Mayday (MJM)-adding value to existing operational tests [CD-ROM]. In Proceedings of the ITS America Seventh Annual Meeting (Washington, DC: ITS America).

Spelt, P., 1997, Development and evaluation of an in-vehicle information system [CD-ROM]. In Proceedings of the ITS America Seventh Annual Meeting (Washington, DC: ITS America).

Srinivasan, R. and Jovanis, P., 1997, Effect of selected in-vehicle route guidance systems on driver reaction times. Human Factors, 39, $200-215$.

Sternberg, S., 1969, The discovery of processing stages. In W. C. Koster (ed.), Attention and Performance, vol. 2 (Amsterdam: North Holland).

Sugasama, F., Ueno, H., Kaneda, M., Koreishi, J., Shirato, R. and Fukuhara, N., 1996, Development of Nissan's ASV. In Proceedings of the 1996 IEEE Intelligent Vehicles Symposium, Tokyo, pp. 254-259.

Venable, D. L., Machemehl, R. B. and Euritt, M. A., 1995, Electronic Toll Collection Systems (Austin: Center for Transportation Research, Bureau of Engineering Research, University of Texas).

Wieck, M., 1997, FAST-TRAC-a success for dynamic route guidance [CD-ROM]. In Proceedings of the ITS America Seventh Annual Meeting (Washington, DC: ITS America).

Winner, H., Witte, S., Uhler, W. and Lichtenberg, B., 1996, Adaptive Cruise Control System Aspects and Development Trends. Technical Paper 961010 (Warrendale, PA: Society of Automotive Engineers).

Woll, J., 1995, Monopulse Doppler radar for vehicle applications. In Proceedings of the Intelligent Vehicles '95 Symposium (Detroit: IEEE).

Young, S., 1995, Collision avoidance system performance specification for lane-change, merge, and backing: phase I results and future plans. In Proceedings of the Peer Review Workshop of the National Highway Traffic Safety Administration Program (Washington, DC: US DOT, NHTSA). 